Published in final edited form as:

Early Interv Psychiatry. 2013 May ; 7(2): 155-161. doi:10.1111/j.1751-7893.2012.00370.x.

\title{
Treatment Possibilities for Individuals at Clinical High Risk of Psychosis
}

\author{
Kali Brummitt ${ }^{1,}$ and Jean Addington ${ }^{2}$ \\ ${ }^{1}$ Department of Psychology, University of Calgary, Alberta, Canada \\ ${ }^{2}$ Hotchkiss Brain Institute, Department of Psychiatry, University of Calgary, Alberta, Canada
}

\section{Abstract}

\begin{abstract}
Aim-The purpose of this study was to undertake a first step in trying to understand the types of treatments young people at clinical high risk of psychosis are interested in pursuing.

Methods-The sample consisted of 30 young people who were current participants in the ongoing North American Prodrome Longitudinal Study (NAPLS 2) and who met criteria for being at clinical high risk for psychosis. Participants were administered a questionnaire that asked them to identify the types of mental health problems they were currently getting help with or would like to get help with and the types of treatments they thought would be helpful and may be interested in receiving.
\end{abstract}

Results-Results showed that when individuals first began participation in NAPLS, almost half had no expectations for treatment. They wanted help but they did not really know what kind of help to be asking for. Participants had a wide range of concerns for which they wanted help. Although a majority endorsed attenuated positive symptoms as a concern, many other issues such a family and social problems were often identified. For those who were already receiving help, the general opinion was that it was beneficial.

Conclusions-The findings suggest that this population sees many different interventions as being potentially helpful. Furthermore, they are open to participating in a variety of treatments, including psychotherapeutic and psychiatric interventions as well as more general treatments addressing a range of possible deficits or difficulties.

\section{Keywords}

early intervention; schizophrenia; ultra-high risk for psychosis; treatment; client participation

\begin{abstract}
One of the major goals of studying those at risk of developing psychosis is determining the level of risk of conversion to a full blown psychotic illness and identifying predictors of conversion $^{1-3}$. Criteria have been established for identifying these young people who are considered to be at "clinical" or "ultra" high risk for developing psychosis ${ }^{4,5}$. This paper will use the term clinical high risk (CHR). Individuals who meet CHR criteria report a diverse array of symptoms and concerns and the potential benefits of treatment for these young people have been recognized for some time. As Woods et al. ${ }^{6}$ stated, these individuals are, "symptomatic, functionally impaired, and [in some cases] treatmentseeking" (p. 200). It is important to note, however, that while these symptoms may precede frank psychosis, conversion to psychosis is not certain. Many people who present with some
\end{abstract}

\footnotetext{
*Corresponding Author: Kali Brummitt, kalibrummitt@hotmail.com.
} 
or all of these symptoms will not progress to psychosis ${ }^{7}$. Regardless an important question is what might be the best treatment to offer such a diverse group of young people.

A range of treatment studies have suggested that intervention before a potential first episode of psychosis may possibly delay the onset of psychosis and may improve symptoms. The first randomized controlled trial (RCT) reported the effectiveness of a combination of antipsychotics and a psychological treatment in reducing conversion. ${ }^{8}$ The second RCT compared the effectiveness of anti-psychotics to placebo and although the rate of conversion was reduced by $50 \%$ this was not significant. ${ }^{9}$. However, there have been some concerns such as metabolic side effects or whether we know enough about the impact of antipsychotics with respect to the use of antipsychotics in this at risk population ${ }^{10}$. Other RCTs looking at the effectiveness of psychological interventions, in particular cognitive behaviour therapy (CBT) reported a reduction in positive symptoms ${ }^{11,12}$, enhanced social adjustment ${ }^{13}$, improvements in anxiety and depression ${ }^{12}$, and, in some cases, a significantly lower risk of progressing to psychosis ${ }^{11}$. Furthermore, some of these benefits persisted at a 3 -year follow-up ${ }^{15}$. A unique study conducted by Amminger et al. ${ }^{14}$ demonstrated a significant impact on symptoms and functioning and a reduced conversion rate in those at CHR who received long-chain omega-3 polyunsaturated fatty acids compared to placebo.

Regardless of the type, treatment can benefit those at risk of developing a psychotic disorder. Even amongst those diagnosed as CHR who do not go on to develop psychosis, their symptoms are distressing and disabling. It can be argued that these individuals would also benefit from treatment that might improve symptoms and functioning. Some of the studies discussed above posit that even those who convert to psychosis benefit from regular contact with mental health professionals ${ }^{8,9}$.

In these young CHR individuals, potential side effects and stigma associated with participating in treatment need to be considered. In response to concerns about what treatments to offer, McGorry and colleagues ${ }^{16}$ suggest employing a clinical staging model in which the intensity of treatment is matched to the stage and severity of the psychiatric disorder. Although the issue of acceptability of certain treatments for those at CHR has been raised by some researchers ${ }^{15}$, to our knowledge no studies to date have actually addressed this issue.

The purpose of this study was to undertake a first effort to gain insight into the acceptability of treatments for these CHR young people. By acceptability we mean interested in pursuing for their particular problems. It is intended to determine what kinds of mental health treatments people at risk of developing a psychotic disorder think may be helpful to them and whether they would be interested in participating in these treatments if they were available. To the best of our knowledge, this is the first study to ask this population about the types of interventions they believe would be helpful. While many of the treatments currently being offered are interventions already in use for individuals experiencing frank psychosis, the at-risk population may have unique needs and further exploration of the range of interventions that could be offered must be explored.

\section{Methods}

\section{Participants}

A sample of convenience was gathered from participants at the Calgary site of the ongoing North American Prodrome Longitudinal Study (NAPLS 2), an 8 site collaborative two year study of individuals at clinical high risk of psychosis that is examining clinical and biological predictors of conversion to psychosis. Recruitment for NAPLS participants had been sought from a variety of sources including family physicians, student counsellors, and 
community mental health teams and practitioners. Sixty-eight participants are currently enrolled in the Calgary NAPLS 2 project, 30 of whom participated in this study.

This sample met the inclusion and exclusion criteria for the NAPLS 2 project. Inclusion criteria consisted of being between 12 and 35 years old and meeting criteria for one of the three prodromal syndromes ${ }^{17}$ using the Structured Interview for Prodromal Syndromes (SIPS). Exclusion criteria consisted of (i) meeting criteria for a current or lifetime Axis I psychotic disorder, including affective psychoses and psychosis NOS; (ii) no current treatment with antipsychotic medication unless it can be clearly demonstrated that the diagnostic prodromal criteria were present prior to the antipsychotic; (iii) impaired intellectual functioning, (i.e. IQ<70); (iv) past or current history of a clinically significant central nervous system disorder that may contribute to prodromal symptoms or confound their assessment; (v) traumatic brain injury that resulted in loss of consciousness, and/or (vi) or a serious alcohol or drug dependence in the last 3 months.

All participants gave written consent prior to the study. Parental consent was obtained from participants under 18 years. Study procedures were explained to all participants.

\section{Measures}

The data collection measure was specifically designed to explore what kind of treatment CHR individuals think may be helpful for them. The measure was developed through consultation with professionals who had expertise in working with CHR young people, in their presenting concerns, in the assessment of those at CHR, and in both biological and psychosocial treatments for those at different phases of a psychotic illness. The questionnaire centred around the types of mental health problems participants were currently getting help with or would like to get help with and the types of treatments they might be interested in participating in, if they were to be offered.

The questionnaire focuses on five distinct areas. 1. Mental health issues with which the participant is currently receiving help. These include anything the participant presents, but typically focuses on symptoms related to the CHR diagnosis. This section begins with an open ended question, followed by a list of specific mental health issues for which they may be getting help. 2. The participant rates their current treatment or support, if any, on a Likert-like scale. 3. An open-ended question asks what type of help the participant was expecting when they began participating in the NAPLS project. 4. This section asks what, if any, mental health issues the participant would like to get help with, but is not currently. This is addressed by asking an open-ended question followed by a specific list of mental health issues with which they may want help. 5 . The final section focuses on several common forms of treatment for mental illness. Treatments include psychological, supportive and medical. Brief descriptions of each treatment are provided and the participant is asked whether they think each treatment would be helpful for them, whether they would take part in the treatment if it were being offered, and why or why not.

\section{Procedure}

Thirty-one participants were invited to participate in this study and 30 agreed to participate. Since this was a sample of convenience participants were invited to participate when they came in for a follow-up assessment. Questionnaires were conducted as interviews and typically took 30 to 60 minutes to complete. Interviews were conducted by KB or one of the research raters for the NAPLS project. Participants were recruited when they were attending for one of their follow-up assessments or when they had completed all of the baseline assessments. 


\section{Analysis}

Frequencies of endorsing presenting problems and specific treatment choices are presented using percentages. Presenting problems were collapsed into groups of symptom categories. For example, feeling suspicious, unusual thoughts or ideas, perceptual changes, and disorganized thinking or speaking were put into a Positive Symptoms category. Symptom categories were then cross-tabulated with treatment endorsements to assess whether those wanting help for a particular category of symptom endorsed one treatment more frequently than others. Open-ended answers regarding treatment expectations were coded and put into eight categories.

\section{Results}

The average age of the sample ( 21 males, 9 females) was 19.1 years ( $\mathrm{SD}=4.45$, range $14-35$ years). All but one were single (96.7\%), and a third were currently employed (33.3\%). The sample had an average of 11.8 years of education $(\mathrm{SD}=2.52)$ and $22(73 \%)$ were currently students. Approximately half had not yet finished high school (56.7\%), nine had completed high school (30.0\%) and four (13.4\%) others had completed some type of post-secondary education.

In response to what mental health issues participants were currently getting help with, more than half reported stress $(56.7 \%)$ and/or depression $(53.0 \%)$. Nearly half of the participants reported trouble concentrating (46.7\%), anxiety (40.0\%), sleeping problems (40.0\%), and/or lack of motivation (40.0\%). Notably, no participants reported getting help for sexuality, sexual orientation, or gender issues, although two participants reported that they would like help with one of these issues. Other concerns participants reported included disorganized thinking or speaking (33.3\%), anxiety (26.7\%), depression (23.3\%), and irritability and/or anger (23.3\%). Details of the presenting problems and concerns are presented in Table 1.

In response to treatment expectations when they first sought help, almost half (46.7\%) had no clear expectation of the type of help or treatment they would receive and a further two individuals (6.7\%) specified a particular issue that they wanted help with (e.g. depression), but did not specify what type of help they expected. Eleven people (36.7\%) anticipated some type of therapy or counselling and eight participants (26.7\%) expected information or guidance about their symptoms, or a psychiatric diagnosis. On the other hand, only one participant (3.3\%) expected to meet with a psychiatrist, while two people were expecting medications (6.7\%) and another participant (3.3\%) specifically stated that they did not want to be prescribed medications. Of the 30 participants, only one (3.3\%) felt that they did not need any help and so expected none.

When a list of common psychological treatments was given to participants, individuals often endorsed more than one potential therapy as seeming helpful, with mean number of items endorsed of 9.5 and a range of 2-16. Treatments most frequently endorsed as being helpful included seeing a psychiatrist (86.7\%), CBT (80.0\%), psychiatric medications $(80.0 \%)$, ST (76.7\%), and a third (66.7\%) thought psychoeducation would be helpful. Participants generally were also open to participating in a range of treatments. The number of treatments endorsed per participant ranged from 1-13 and the mean was 6.0. The most common treatments participants reported they would likely participate in were CBT (63.3\%) and psychoeducation (63.3\%). Furthermore, nearly half would be willing to participate in interpersonal psychotherapy (46.7\%) and/or cognitive remediation (46.7\%). Details of the treatments participants endorsed as helpful and would likely participate in are presented in Table 2. 
Spearman's correlations between age and treatment endorsement were examined. Although age did not have an impact on the number of treatments participants thought would be helpful, there was a significant positive correlation between age and the number of treatments participants reported they would participate in, $r(28)=0.36, p<0.05$. Therefore, older participants were more willing to engage in a greater range of treatments.

Treatment endorsements were examined according to the types of symptoms with which participants would like help. Among those who wanted help with positive symptoms, the treatment in which participants most frequently reported they would take part was supportive group therapy. Participants wanting help with negative symptoms as well as those wanting help with other psychological issues most often endorsed CBT. Participants who wanted help with cognitive issues and those who wanted help with social issues both endorsed psychoeducation most frequently as a treatment in which they would likely participate. Details of these results are presented in Table 3.

\section{Discussion}

To the best of our knowledge this is the first study to ask young people at CHR for psychosis what concerns they felt they needed help with and about the types of interventions they believe would be helpful to them and would likely participate in, if they were available.

When individuals first sought help, they often had a wide range of concerns such as attenuated positive symptoms and family and social problems that they wanted addressed. Although many had ideas about what kind of help they might want, almost half had no expectations for treatment. For those who were already receiving some help, the general opinion was that it was beneficial.

The findings suggest that this population sees many different interventions as being potentially helpful. Furthermore, they are open to participating in a variety of treatments, including psychotherapeutic and psychiatric interventions as well as more general treatments addressing a range of possible difficulties. What is most interesting and relevant is that participants generate a wide range of concerns for which they wanted help, yet they really did not know what kind of help they might like. Once introduced to several types of help, they made an effort to try and identify what would most likely be helpful to them. It is also important to note that even if they endorsed a particular treatment as helpful it did not necessarily imply that they would be willing to engage in that treatment. When we consider the treatments endorsed by participants according to the types of issues for which they would like help, some interesting observations were made. For instance, participants wanting help with cognitive difficulties, social issues, and other psychological issues most frequently endorsed seeing a psychiatrist as helpful and participation in psychoeducation as a program in which they would likely participate. These findings might point to their desire for information about their symptoms and a greater understanding of what is happening to them, a sentiment voiced by one participant who said, "[Psychoeducation] could help me understand what's wrong with me. It would be nice to see my problems as symptoms instead of as my fault." In addition, seeing a psychiatrist was the only treatment option that referred to a specific mental health professional and was seen by some as a trustworthy and accurate source of information and guidance. As one participant put it, "[Psychiatrists] can diagnose a problem. They're somebody you can trust that's making the right decisions for you."

Participants seeking help for positive symptoms on the other hand, endorsed supportive group therapy most often, both for its perceived helpfulness and as a treatment in which they would likely participate. It seems that perhaps those coping with subthreshold positive symptoms are seeking to relate to others with similar experiences and to learn about how 
they are managing. This might be due to how unusual and disturbing these symptoms can be. Perhaps these individuals feel the need to normalize what is happening to them. One participant said about possibly participating in supportive group therapy that, "It would reassure you you're not the only one who's going through something and you could get advice." Another's frustrations with feeling isolated can be seen in the following quote: "It would help to meet people in a similar situation who I know understand because I would get less angry because I would know they really do understand."

There are several limitations to this study. First the sample is small. Second, there are proportionally more males which was a function of the sample of convenience being collected as participants came for a follow-up assessments. Third, individuals may not have felt comfortable revealing the range of their symptoms to the interviewer, or may not have had insight into their specific symptoms and mental health issues. Although we offered descriptions of the treatments, generally participants were not that familiar with what the different psychosocial treatments entailed and our descriptions may not have been adequate. However, they did have the opportunity to ask for more details. Finally, some of the participants were receiving some treatments. It was not possible to document any "treatment" they may be receiving we do not know what impact this may have had on their responses. However, our focus was on the kind of help they thought might be useful for their particular concerns.

At this stage in trying to understand and offer intervention for those at clinical high risk of psychosis there is no evidence as to what would be the best treatments. This study demonstrates that these young people are able to identify the problems they think they most need help with and are able for the most part, with help, to identify treatments they think would be helpful. However, although many of the choices made sense, there were times that was not always clear why people made certain choices (e.g. cognitive remediation was not the treatment of choice for cognitive problems, such as attention and memory). This suggests that these young people should play a role in discussions around treatment options. Furthermore, it is most important to address not just what they think would be helpful but to address what they would engage in, as the two are not necessarily the same.

Future research could focus on (i) whether participants' responses in such a study, if given the opportunity to participate in a particular treatment program, are an indication of future behaviour; (ii) an increased understanding of other factors that influence likelihood to participate in a treatment, beyond perceived helpfulness. Interestingly, many participants were interested in psychoeducation and since this has been suggested by McGorry and his colleagues ${ }^{16}$ as a recommended treatment for individuals in the very first stage of the prepsychotic period it may be useful to test this as a first option treatment.

In conclusion, participants found to be at CHR for developing a psychotic disorder perceive many treatments as being potentially helpful and individuals often endorsed more than one therapy. In addition, participants were generally open to participating in a range of treatments, again often endorsing more than one option. The results of this study offer a first step in understanding the types of treatment this population is interested in. It also begins to assess the acceptability of various treatments to the individuals directly involved in them. Future research can now expand on these findings, perhaps eliminating some of the subjectivity by using clinical assessment results and improving on the clarity of the questionnaire or narrowing the focus to just a few of the more popular treatments, allowing for a more in depth examination. Finally, the findings of this study show that when treating young people at risk for psychosis, including them in discussions of planning their own treatment is a viable option and an important opportunity to engage them and better 
understand what issues they would like help with and how they would like to address these concerns.

\section{Acknowledgments}

This work was supported by NIMH grant 1UO1MH081984 to J Addington.

\section{References}

1. Cannon TD, Cadenhead K, Cornblatt B, Woods SW, Addington J, Walker E, et al. Prediction of psychosis in youth at high clinical risk: a multisite longitudinal study in North America. Arch Gen Psychiatry. 2008 Jan; 65(1):28-37. [PubMed: 18180426]

2. Ruhrmann S, Schultze-Lutter F, Salokangas RK, Heinimaa M, Linszen D, Dingemans P, et al. Prediction of psychosis in adolescents and young adults at high risk: results from the prospective European prediction of psychosis study. Arch Gen Psychiatry. 2010 Mar; 67(3):241-51. [PubMed: 20194824]

3. Yung AR, Phillips LJ, Yuen H, Francey S, McFarlane CA, Hallgreen MA, et al. Psychosis prediction: 12-month follow-up of a high-risk ("prodromal”) group. Schizophr Res. 2003 Mar; 60(1):21-32. [PubMed: 12505135]

4. Miller TJ, McGlashan TH, Rosen JL, Somjee L, Markovich PJ, Stein K, et al. Prospective diagnosis of the initial prodrome for schizophrenia based on the structured interview for prodromal syndromes: Preliminary evidence of interrater reliability and predictive validity. Am J Psychiatry. 2002 May.159:863-5. [PubMed: 11986145]

5. Yung AR, McGorry PD, McFarlane CA, Jackson HJ, Patton GC, Rakkar A. Monitoring and care of young people at incipient risk of psychosis. Schizophr Bull. 1996; 22(2):283-303. [PubMed: 8782287]

6. Woods SW, Walsh BC, Saksa JR, McGlashan TH. The case for including Attenuated Psychotic Symptoms Syndrome in DSM-5 as a psychosis risk syndrome. Schizophr Res. 2010 Nov.123:199207. [PubMed: 20832249]

7. Addington J, Cornblatt B, Cadenhead K, Cannon T, McGlashan T, Perkins D, et al. At clinical high risk for psychosis: Outcome for non-converters. Am J Psychiatry. 2011

8. McGorry PD, Yung A, Phillips L, Yuen H, Francey S, Cosgrave E, et al. Randomized controlled trial of interventions designed to reduce the risk of progression to first-episode psychosis in a clinical sample with subthreshold symptoms. Arch Gen Psychiatry. 2002 Oct; 59(10):921-8. [PubMed: 12365879]

9. McGlashan TH, Zipursky RB, Perkins D, Addington J, Miller T, Woods SW, et al. Randomized, double-blind trial of olanzapine versus placebo in patients prodromally symptomatic for psychosis. Am J Psychiatry. 2006 May; 163(5):790-9. [PubMed: 16648318]

10. Bentall RP, Morrison AP. More harm than good: The case against using anti-psychotic drugs to prevent severe mental illness. J Ment Health. 2002 Aug; 11(4):351-6.

11. Morrison AP, French P, Walford L, Lewis SW, Kilcommons A, Green J, et al. Cognitive therapy for the prevention of psychosis in people at ultra-high risk: randomised controlled trial. $\mathrm{Br} \mathrm{J}$ Psychiatry. 2004 Oct.185:291-7. [PubMed: 15458988]

12. Addington J, Epstein I, Liu L, French P, Boydell KM, Zipursky RB. A randomized controlled trial of cognitive behavioral therapy for individuals at clinical high risk of psychosis. Schizophr Res. 2011 Jan; 125(1):54-61. [PubMed: 21074974]

13. Bechdolf A, Wagner M, Veith V, Ruhrmann S, Pukrop R, Brockhaus-Dumke A, et al. Randomized controlled multicentre trial of cognitive behaviour therapy in the early initial prodromal state: Effects on social adjustment post treatment. Early Interv in Psychiatry. 2007 Jan.1:71-8.

14. Amminger GP, Schafer MR, Papageorgiou K, Klier CM, Cotton SM, Harrigan SM, et al. Longchain omega- 3 fatty acids for indicated prevention of psychotic disorders: a randomized, placebocontrolled trial. Arch Gen Psychiatry. 2010 Feb; 67(2):146-54. [PubMed: 20124114] 
15. Morrison AP, French P, Parker S, Roberts M, Stevens H, Bentall RP, et al. Three-year follow-up of a randomized controlled trial of cognitive therapy for the prevention of psychosis in people at ultrahigh risk. Schizophr Bull. 2007 May; 33(3):682-7. [PubMed: 16973786]

16. McGorry PD, Hickie IB, Yung AR, Pantelis C, Jackson HJ. Clinical staging of psychiatric disorders: a heuristic framework for choosing earlier, safer and more effective interventions. Aust N Z J Psychiatry. 2006 Aug; 40(8):616-22. [PubMed: 16866756]

17. McGlashan, T.; Walsh, BC.; Woods, SW. The Psychosis Risk Syndrome: Handbook for Diagnosis and Follow-up. New York, NY: Oxford University Press; 2010. 
Table 1

Presenting Problems and Concerns

\begin{tabular}{|c|c|c|c|}
\hline & $\begin{array}{l}\text { Currently getting help with } \\
\text { this issue, } \mathrm{n}(\%)\end{array}$ & $\begin{array}{l}\text { Want help with this issue, } \\
\text { n(\%) }\end{array}$ & $\begin{array}{c}\text { Do not want help with this } \\
\text { issue, } n(\%)\end{array}$ \\
\hline Positive Symptoms & $18(60.0)$ & $11(36.7)$ & $19(63.3)$ \\
\hline Feeling suspicious & $9(30.0)$ & $3(10.0)$ & $18(60.0)$ \\
\hline Unusual thoughts or ideas & $11(36.7)$ & $6(20.0)$ & $13(43.3)$ \\
\hline Perceptual changes & $10(33.3)$ & $2(6.7)$ & $18(60.0)$ \\
\hline Disorganized thinking or speaking & $3(10.0)$ & $10(33.3)$ & $17(56.7)$ \\
\hline Negative Symptoms & $14(46.7)$ & $10(33.3)$ & $20(66.7)$ \\
\hline Feeling socially isolated & $7(23.3)$ & $4(13.3)$ & $19(63.3)$ \\
\hline Lack of motivation and/or energy & $12(40.0)$ & $6(20.0)$ & $12(40.0)$ \\
\hline Feeling flat or numb & $5(16.7)$ & $5(16.7)$ & $20(66.7)$ \\
\hline Cognitive Difficulties & $16(53.3)$ & $7(23.3)$ & $23(76.7)$ \\
\hline Memory problems & $8(26.7)$ & $5(16.7)$ & $17(56.7)$ \\
\hline Trouble concentrating & $14(46.7)$ & $4(13.3)$ & $12(40.0)$ \\
\hline Trouble solving problem & $8(26.7)$ & $4(13.3)$ & $18(60.0)$ \\
\hline Social Issues & $15(50.0)$ & $13(43.3)$ & $17(56.7)$ \\
\hline Family issues & $9(30.0)$ & $4(13.3)$ & $17(56.7)$ \\
\hline Independent living skills & $4(13.3)$ & $4(13.3)$ & $22(73.3)$ \\
\hline Job functioning & $3(10.0)$ & $4(13.3)$ & $23(76.7)$ \\
\hline School work & $6(20.0)$ & $5(16.7)$ & $19(63.3)$ \\
\hline Social problems & $8(26.7)$ & $4(13.3)$ & $18(60.0)$ \\
\hline Other Psychological Issues & $25(83.3)$ & $14(46.7)$ & $16(53.3)$ \\
\hline Depression & $16(53.3)$ & $7(23.3)$ & $7(23.3)$ \\
\hline Anxiety & $12(40.0)$ & $8(26.7)$ & $10(33.3)$ \\
\hline Stress & $17(56.7)$ & $6(20.0)$ & $7(23.3)$ \\
\hline Trouble sleeping or sleeping too much & $14(46.7)$ & $6(20.0)$ & $10(33.3)$ \\
\hline Irritable and anger & $7(23.3)$ & $7(23.3)$ & $16(53.3)$ \\
\hline Suicide and/or self-harm & $9(30.0)$ & $3(10.0)$ & $18(60.0)$ \\
\hline Self-esteem or self-confidence issues & $9(30.0)$ & $6(20.0)$ & $15(50.0)$ \\
\hline Sexuality, sexual orientation or gender issues & $0(0.0)$ & $2(6.7)$ & $28(93.3)$ \\
\hline Alcohol and/or drugs use & $3(10.0)$ & $0(0.0)$ & $27(90.0)$ \\
\hline Trauma & $6(20.0)$ & $4(13.3)$ & $20(66.7)$ \\
\hline
\end{tabular}

Early Interv Psychiatry. Author manuscript; available in PMC 2014 May 01. 


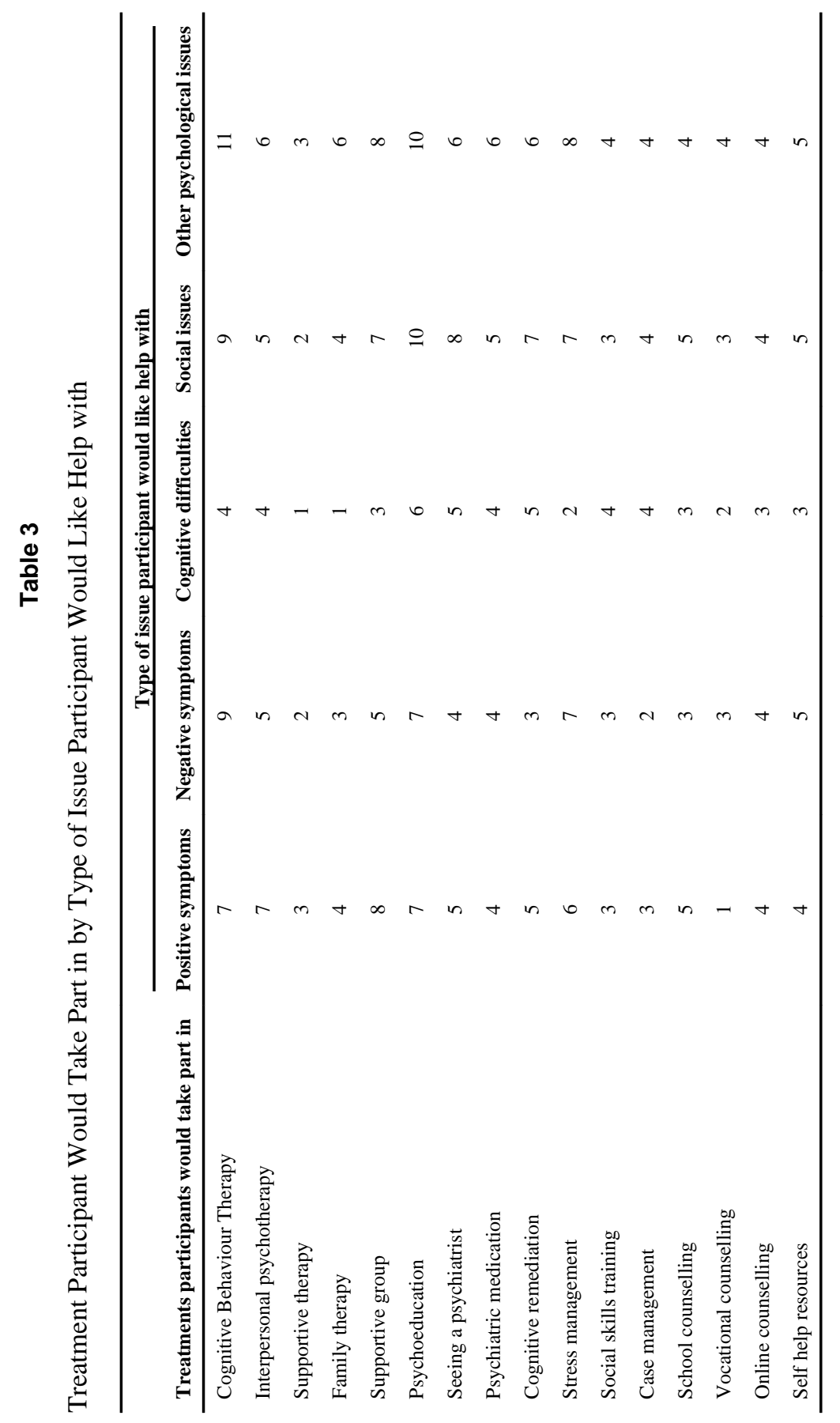

Early Interv Psychiatry. Author manuscript; available in PMC 2014 May 01. 